\title{
Diabetic Mastopathy, Diagnoses and Management
}

\section{Ragmoun $\mathrm{H}^{1 *}$, Ajili $\mathbf{A}^{1}$ and Daadoucha $\mathrm{A}^{2}$}

${ }^{1}$ Department of Obstetric Gynecology, Ibn El Jazzar Hospital Kairouan, Kairouan 3100, Tunisia

${ }^{2}$ Department of Radiology, Ibn El Jazzar Hospital Kairouan, Kairouan 3100, Tunisia

\begin{abstract}
Diabetic mastopathy (DM) is a pathological entity of recent description, it is rare and benign; occurring in young patients with type I diabetes, or having an autoimmune disease. Clinically, she simulates breast cancer. Radiological examinations are not very informative. The diagnosis of certainty is histological. Through an observation of (DM) occurred in a diabetic patient, under insulin for 14 years and carrying multiple degenerative complications, we recall the clinical aspects, radiological and evolutionary of this affection.
\end{abstract}

Keywords: Mastopathy; Type 1 diabetes; Breast cancer

\section{Introduction}

The diabetes mellitus that has been developed for a long time is causes of various complications, affecting most organs. For a long time it has been thought that the mammary gland is safe from these complications. It was not until 1984 that Soler and Khardori [1] described for the first time a case of breast lesions associated with old diabetes. The term of diabetic mastopathy (DM), commonly used is introduced by Tomaszewski et al. [2]. These (DM) are rare, benign and occurring more particularly in patients with of an old type I diabetes. They take on the appearance of a tumor lesion simulating breast cancer both clinically and radiologically. We report a new case from (DM) in the light of which we discuss the problems of diagnosis and management of this condition.

\section{Case Report}

Miss A.D., age 33, single, with no background breast cancer patients, followed for 14 years for type I diabetes under insulin therapy, her diabetes is unbalanced, at the origin of many episodes of metabolic decompensations and source of multiple degenerative complications: diabetic retinopathies treated by laser since four years; kidney failure on dialysis since 2013. Hypertensionunder adalate $\mathrm{LP}^{\star}$ since five years. Moreover it makes iterative infiltrations for a left. scapulohumeral periarthritis. She consults us in December 2015 for a left breast nodule of recent appearance (three months). Examination found at the inspection: two symmetrical breasts, no morphological abnormalities in particular not nipple retraction or orange peel appearance or cutaneous redness.

On palpation, there is poorly limited tumor formation of 3 to $4 \mathrm{~cm}$ in diameter, of stony consistency, painless, mobile compared to plans shallow and deep and sitting at the union of the quadrants. There is no flow mammary or axillary lymphadenopathy. Examination of the right breast is normal. The Mammography find dense type IV breasts of BIRADS without microcalcification and architectural distortion (Figure 1).

Ultrasound finds a poorly limited hypoechoic formation of $2.15 \mathrm{~cm}$ in diameter with acoustic attenuation but without doppler vascular flow. The lesion is classified ACR 4 (Figure 2). The mammary cytopuncture resulted in a sample insufficient cell with some ductal cells without atypia.

An excisional biopsy of the tumor was performed. The latter is made of hard and dystrophic breast tissue. Macroscopically the biopsy fragment measures $4 \times 3 \times 3 \mathrm{~cm}$ made of very firm fibrous tissue. Histological examination showed that the lesion consists of a stroma dense fibrous tissue, with periobular pericanal and perivascular lymphocyte infiltration. This lymphocyte infiltration is without signs of malignancy, as well as the presence fusiform fibroblastic cells (Figures 3 and 4). Aspect evoking a fibrous mastopathy of the breast, the immediate operative follow-ups are simple, the medium-term trend was marked by the recurrence of breast nodule at the same location within four month for which she benefited from a new surgical excision and pathological examination confirming the fibrous nature of this mastopathy.

\section{Discussion}

DM also called fibrous breast disease [1] or Sclerosing lymphocytic lobulitis $[3,4]$ is an entity recent pathological, rare and benign; about

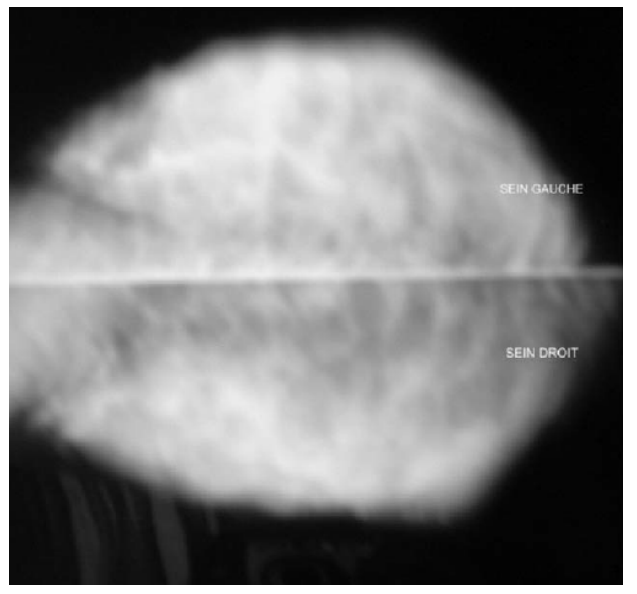

Figure 1: Mammography: denses breasts type IV of BIRADS without microcalcification and architectural distortion.

*Corresponding author: Houssem R, Department of Obstetric Gynecology, Ibn EI Jazzar Hospital Kairouan, Ibn El Jazzar Street, Kairouan 3100, Tunisia, Tel: +216 98451 341; E-mail: houssem.ragm@yahoo.fr

Received October 05, 2017; Accepted November 06, 2017; Published November 16,2017

Citation: Ragmoun H, Ajili A, Daadoucha A (2017) Diabetic Mastopathy, Diagnoses and Management. J Cytol Histol 8: 485. doi: 10.4172/2157-7099.1000485

Copyright: (c) 2017 Ragmoun $\mathrm{H}$, et al. This is an open-access article distributed under the terms of the Creative Commons Attribution License, which permits unrestricted use, distribution, and reproduction in any medium, provided the original author and source are credited. 
Citation: Ragmoun H, Ajili A, Daadoucha A (2017) Diabetic Mastopathy, Diagnoses and Management. J Cytol Histol 8: 485. doi: 10.4172/21577099.1000485

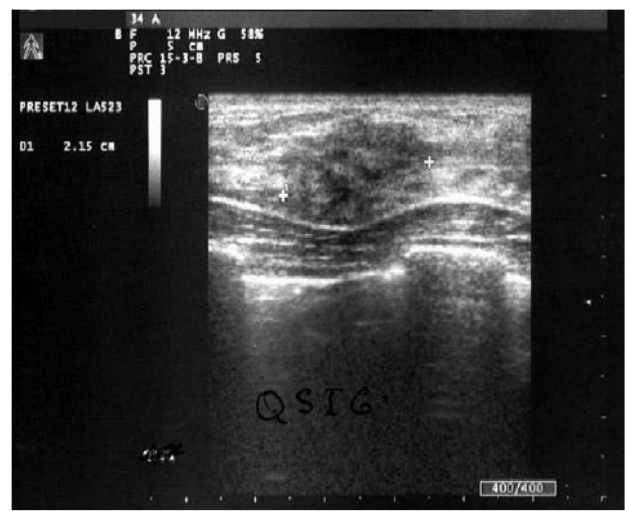

Figure 2: Ultrasonography: ill-definited margins masses with marked hypoechogenicity and posterior acoustic shadowing measuring $2.5 \mathrm{~cm}$ in diameter.

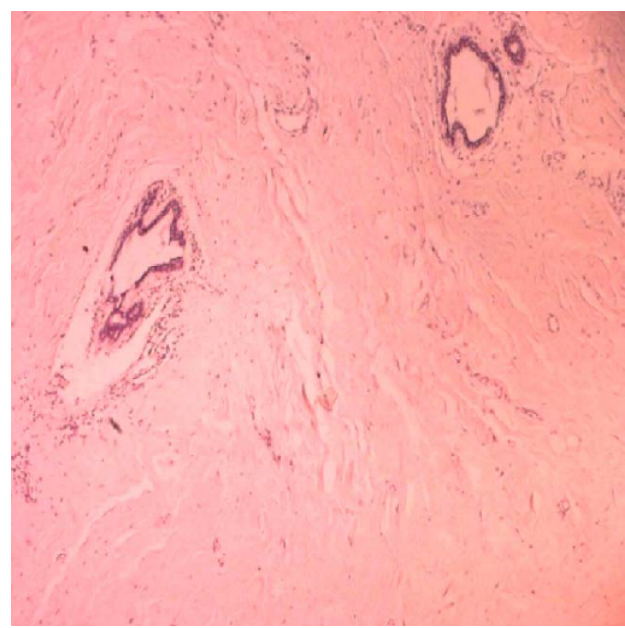

Figure 3: Several lobules have lymphocytic infiltration without evidence of malignancy.

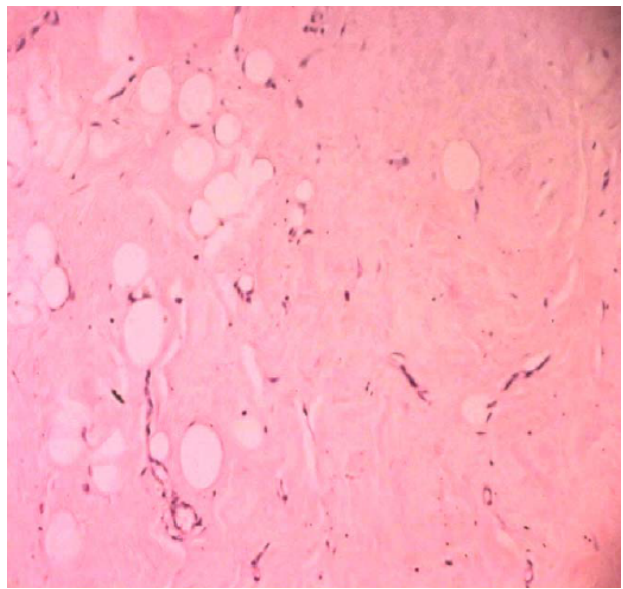

Figure 4: Sclerosing mammary tissue, lymphocytic lobulitis, which shows atrophic changes.

200 cases are reported in the literature. The prevalence of this disease is unknown because of the ignorance of the affection and the publications reported only sporadic cases. The frequency of occurrence of these
(DM) is estimated at $0.06 \%$ according to Logan and $13 \%$ for Soler and Khardori [1]. According to Hunfeld and Bassler [5], the (DM) represents less than $1 \%$ of all the benign lesions of the breasts, this rate rises to $13 \%$ only for Type I diabetic patients.

This condition mainly affects young women with type I diabetes, often at the stage of degenerative complications [6]. More rarely same anatomical lesions have been described in other autoimmune diseases (thyroiditis, lupus) or diabetes type II insulin requiring [1,7]. Bilaterality is found in about $63 \%$ of cases [8]. Male involvement is exceptionally, 5 cases have been reported in diabetic men [6,7]. Our patient is type I diabetic, carrier retinal degenerative complications, renal and osteo articular.

The pathophysiology of (DM) is not totally elucidated. It would be multifactorial and many assumptions have been advanced:

- Hyperglycemia is thought to be the cause of an accumulation of abnormal and decreased conjunctiva tissue degradation in the extracellular matrix $[2,3,8]$.

- Many authors $[3,7,8]$ evoke the hypothesis of a immune reaction, secondary to excess glycosylation, involving lymphocyte $\mathrm{B}$ cells and macrophages; the presence of cytokines would accentuate the Collagenization phenomenon. The presence of mast cells could promote the secretion of collagen VIII.

Some authors suggest possible toxicity to exogenous insulin. Seidman et al. [9] reported cases of (DM) in type II diabetic patients becoming Insulino necessitantes. They incriminate exogenous insulin in the genesis of these (DM) through an inflammatory or immune reaction. Clinically, the (DM) constantly presents itself as a single, or multiple, uni or bilateral breast nodule, of recent appearance, irregular contours, consistency hard, even stony, painless, often growing fast, mobile compared to both shallow and deep variable, but frequently retro-areolar measuring 0.5 to $6 \mathrm{~cm}$ long axis. It is not associated with a nipple flow nor to inflammatory phenomena skin. These lesions simulate clinically breast cancer and it is difficult to distinguish these two entities by the simple physical examination. It is for this reason that recourse to additional exams or surgery is essential.

Radiological examinations provide little information; so the mammograms show frequently overdensities, opacities more or less regular, even dense breasts but without architectural distortion or micro calcification foci $[7,8]$.

On ultrasound, the aspects are variable. Typically, the lesion results in a hypoechoic formation, more or less irregular contours responsible for a marked attenuation of ultrasound $[4,8,10]$. These anomalies ultrasound were found in our patient. Breast MRI has been tested in limited cases; but it does not differentiate the (DM) from a possible breast cancer $[3,10]$.

According to the majority of the studies consulted, radiological examinations are not very reliable and not very specific to this pathology, histological examinations are therefore always essential for a definitive diagnosis.

Fine needle cytopoints are difficult to achieve because of the sclerous nature of the lesion. Williams et al. [4] and Logan report a $50 \%$ rate of punctures uninterpretable because of their cellular material poverty. Gump and Mc Demott met the same difficulties; in fact, out of a total of nine cytological punctures, seven are acellular and the other two cases are very poor in cells to allow interpretation. 
Citation: Ragmoun H, Ajili A, Daadoucha A (2017) Diabetic Mastopathy, Diagnoses and Management. J Cytol Histol 8: 485. doi: 10.4172/21577099.1000485

In the rare cases where the cytological puncture contains cellular material there is often an abundant fibrous tissue hyalinized. This fibrous tissue is about ten times more abundant than in normal epithelial tissue. Cytological puncture in our case was very poor in cells and considered uninterpretable. The biopsy is the unavoidable examination that allows the diagnosis.

The histological study highlights three kinds of elementary, nonspecific, variously associated lesions:

- Lymphocyte lobulitis: mononuclear inflammatory infiltrate, more or less dense, associating small lymphocytes especially B, plasma cells and histiocytes. It sits primarily in the breast lobules but also and less marked in perivascular and sometimes in peri-canalar;

- A fibrosis of the pallial tissue, always found, is homogeneous and dense;

- Inconsistently, stromal epithelioid cells homogeneously distributed;

Immunohistochemistry has no diagnostic indication. It can be useful in differential diagnosis with:

- Invasive lobular carcinoma (anti-keratin antibodies) in cases where epithelioid cells are particularly many $[3,8]$.

- Low-grade non-Hodgkin's lymphoma when lymphoid infiltrate appears suspect [7].

After surgery excision; evolution is marked by a high rate of recurrence of lesions ranging from $20 \%$ according to Hunfeld and Bassler [6] to $100 \%$ according to Lammie [3]. Our patient presented rapid recurrence after four months of the procedure. A new tumor excision is practiced.

By the way, when the diagnosis is made by microbiopsy, radical surgery is not necessary. A Simple monitoring is recommended, it includes an annual clinical examination and a mammogram both years, it is possible that the (DM) affects a healing process [8]. No direct relationship between (DM) and breast cancer has been reported in the literature and the risk of developing breast cancer later joined that of the general population $[3,7]$.

\section{Conclusion}

Diabetic mastopathy is a rare condition that is benign mainly occurring in young patients with type I diabetes, having multiple degenerative complications. It simulates clinically breast cancer. Mammography is informative and the diagnosis of certainty is based on histological examination. Its evolution is dominated by risk of recurrence. After exclusion of a neoplastic pathology by microbiopsy, its management is limited to a simple surveillance.

\section{Authors' Contributions}

R.H: Editing, supervision, read and approved the final manuscript. D.A: participated in the drafting of the observation, read and approved the final manuscript. B.N: participated in the drafting of the discussion, read and approved the final manuscript. A.A: checking references.

\section{Acknowledgements}

We thank the Anatomopathology Department of Ibn El Jazzar Hospital Kairouan.

\section{References}

1. Soler NG, Khardori R (1984) Fibrous disease of the breast, thyroiditis and cheiroarthropathy in type 1 diabetes mellitus. Lancet 1: 193-195.

2. Tomaszewski JE, Brouks JS, Hicks D, Li Volsi VA (1992) Diabetic mastopathy: a distinctive clinicopathologic entity. Hum Pathol 23: 780-786.

3. Bouté V, Denoux Y, Feilel V, Lemery S, Lacroix J, et al. (2003) Mastopathie diabétique : complication peu commune du diabète de type I. J Le Sein 13 261-269.

4. Williams PH, Rubin CM, Theaker JM (1995) Sclerosing lumphocytic lobulitis of the breast. Clin Radiol 50: 165-167.

5. Hunfeld KP, Bassler R (1997) Lymphocytic mastitis and fibrosis of the breast in long-standing insulin-dependent diabetics. A histopathologic study on diabetic mastopathy and report of ten cases. Gen Diagn Pathol 143: 49-58.

6. Kudva YC, Reynolds CA, O’Brien T, Crotty TB (2003) Mastopathy and diabetes Curr Diab Rep 3: 56-59.

7. Hadj Ali I, Ben Abdallah N, Khiari K, Ben Salem L, Cherif L, et al. (2002) Mastopathie fibreuse diabétique à propos d'un cas. Ann Endocrinol 63: 235-239.

8. Murakami R, Kumita SI, Yamaguchi K, Ueda T (2009) Diabetic mastopathy mimicking breast cancer. Clin Imaging 33: 234-236.

9. Seidman JD, Schnaper LA, Phillips LE (1994) Mastopathy in insulin-requiring diabetes mellitus. Hum Pathol 25: 819-824.

10. Andrews-Tang D, Diamond AB, Rogers L, Butler D (2000) Diabetic mastopathy: adjunctive use of ultrasound and utility of core biopsy in diagnosis. Breast $\mathrm{J} 6$ : 183-188. 